\title{
Optimization and Comparisons for Separation, Detection and Quantification of 12 Aminoglycosides Using 2 Chromatographic Conditions by LC-MS/MS
}

\author{
Samia Mokh ${ }^{1}$, Farouk Jaber ${ }^{1}$, Abir Kouzayha', Hélène Budzinski², \\ Mohamad Al Iskandarani ${ }^{1,3 *}$ \\ ${ }^{1}$ Laboratory for Analysis of Organic Compound (LACO), Lebanese Atomic Energy Commission (LAEC), National \\ Council for Scientific Research (CNRS), Beirut, Lebanon \\ ${ }^{2}$ ISM-LPTC-UMR 5255 (Laboratory of Physico and Toxico Chemistry), CNRS, Bordeaux University 1, Talence, \\ France \\ ${ }^{3}$ Faculty of Public Health, Lebanese University, Beirut, Lebanon \\ Email: mohamad.iskandarani@cnrs.edu.Ib
}

Received 17 August 2014; revised 25 September 2014; accepted 20 October 2014

Copyright @ 2014 by authors and Scientific Research Publishing Inc.

This work is licensed under the Creative Commons Attribution International License (CC BY).

http://creativecommons.org/licenses/by/4.0/

(c) (i) Open Access

\section{Abstract}

Aminoglycosides are a family of antibiotics with important applications in veterinary medicine. Their ionic character, the similarity structures and the high polarity due to the presence of two or more amino and hydroxyl groups cause a difficulty in separation and make these compounds poorly retained on the reversed phase column. An analytical method for the separation and detection of 12 aminoglycosides has been optimized using two kinds of chromatographic conditions (HILIC, Ion pairing). In Hydrophilic Interaction, ZIC_HILIC column was used, by which the following parameters for the mobile phase were evaluated: concentration of ammonium acetate buffer, percentage of formic acid and effect of acid type. The maximum and adequate concentration of ammonium acetate for the majority of analytes was set to $30 \mathrm{mM}$. The percentage $0.1 \%$ of formic acid increases the response for the majority of analytes. On the other side, the use of $0.1 \%$ of trifluoroacetic acid improves the response when compared with the response obtained with $0.1 \%$ of formic acid except for Spectinomycin Dihydrostreptomycin and Streptomycin. For ion pairing chromatography, the concentration of pentafluoropropionic acid was tested and the greatest value appeared to be $9.2 \mathrm{mM}$. Therefore, the comparison between the two separation methods shows that the response area of the majority of analytes tested increases when using the ion pair mode. Also, the high value of $\mathrm{S} / \mathrm{N}$ and the lower detection limit (5 - $15 \mu \mathrm{g} \cdot \mathrm{mL}^{-1}$ ) for most aminoglycosides studied make the ion pairing method more preferable than HILIC interaction.

\footnotetext{
"Corresponding author.
}

How to cite this paper: Mokh, S., Jaber, F., Kouzayha, A., Budzinski, H. and Al Iskandarani, M. (2014) Optimization and Comparisons for Separation, Detection and Quantification of 12 Aminoglycosides Using 2 Chromatographic Conditions by LC-MS/MS. American Journal of Analytical Chemistry, 5, 982-994. http://dx.doi.org/10.4236/ajac.2014.514105 


\section{Keywords}

\section{Aminoglycosides, Ion Pairing, ZIC-HILIC, LC-MS/MS}

\section{Introduction}

Aminoglycosides (AGs) are a large class of antibiotics that are characterized by two or more amino sugars linked by glycosidic bonds to an aminocyclitol component (Figure 1) [1].

They have been widely used in veterinary medicine and animal husbandry and they are distributed in the body after injection where little amount is absorbed from the gastro-intestinal tract. Thus, they are excreted unchanged in the urine [2]. Most of AGs are used against bacteria and parasites in the production of pork, chicken, beef, milk and eggs around the world, which could arouse side reactions and antimicrobial resistance to consumers [3]. These AGs interfere with bacterial protein synthesis by binding irreversibly to ribosome's, in order to interrupt the protein synthesis, which causes damage to the cell membranes [4].

Due to their toxicity and possible antibiotic resistance, considerable attention has been paid to the potential human health risk. Hence, the European Union (EU), the USA, China, Japan, and other countries have issued strict maximum residue levels (MRLs) for nine AGs in various animal origin foods [5]. To monitor these residues in live animals and animal products, two legislations settings are given in Council Directive 96/23/EC5, S.I. 507/98 and EU Commission Decision 2002/657/EC6. Even though, they are banned as growth promoters in the EU [6].
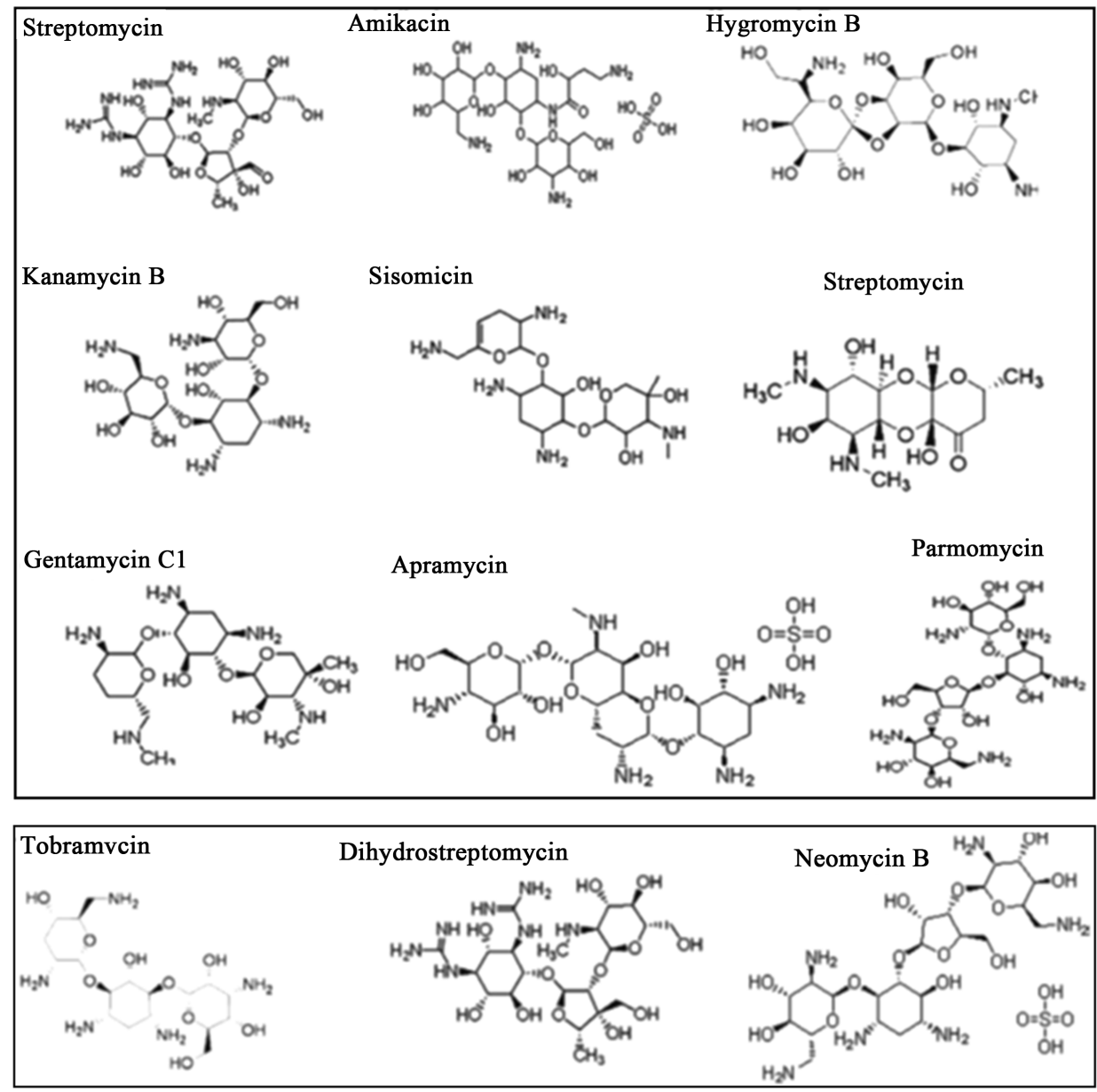

Figure 1. Chemical structures of the 12 AGs. 
Consequently, there is a great need to develop sensitive and reliable analytical methods for monitoring the residues of trace level AGs in complex matrices. Several analytical methods have been described in literature for determination of AGs in biological samples (plasma, urine, milk...) [7]-[10], edible tissues (kidney, muscle...) or varieties of water samples (Hospital water, wastewater....) [11].

AGs are characterized by thermal stability and non-volatility, requiring long derivatization time using $G C$ or GC-MS [12]. Many authors have overcome the problem of the lack of $U V$ chromophore or fluorophore for the AGs by using derivatizing agents for the detection by ultraviolet absorbance (UV) or by fluorescence (FLD) [13]. In this field, the analysis by liquid chromatography $(L C)$ often entails pre- or postcolumn derivatization, followed by the detection with $U V$ or FLD [12] [14]-[16]. However, the derivatization steps render the analytical process moretime consuming and may even introduce impurities. Another problem associated with derivatization is the possibility of self degradation of these derivatives few hours after their formation. This leads to a poor reproducibility caused by their instability and a low yield of derivatives [17]. Therefore, high-performance liquid chromatographic (HPLC) methods in combination with tandem mass spectrometry may have the greatest potential for accomplishing multi-residue identifications in complex matrices [18]. For AGs ionization in MS, the analysis depends on two main types of atmospheric pressure ion sources: the electrospray ionization (ESI) and the atmospheric pressure chemical ionization (APCI). In fact, the ESI is the most commonly used and few publications relied on the APCI for AGs ionization by LC-MS [19] [20].

Sensitivity and selectivity related problems have prompted several researchers to use liquid chromatography coupled to tandem mass spectrometry (LC-MS/MS) to analyze AGs [5] [15] [18]. AGs are well known for their high polarity, thus, they are not retained on the reversed-phase columns [5] [21]. This fact presents a big analytical challenge. So, there were numerous methods to produce some retention of the chromatographically unretained AGs using either ion pairing agents or hydrophilic interaction chromatography which is recently cited by researchers but no explanation was found for their choice [5] [21]-[23]. Our paper contributes to a better understanding of both, the advantages and disadvantages of these two methods HILIC and Ion pairing.

The main objective of our paper is to develop and optimize a reliable and generic $L C$-MS/MS method in order to quantify 12 AGs in water. The originality of our work is related to the presentation and discussion of the potential factors affecting response and sensitivity using ion pairing or HILIC for separation.

\section{Experimental}

\subsection{Materials, Chemicals and Reagents}

Apramycin sulphate (APR) (purity 98.5\%), Gentamycin-2.5-sulphate hydrate (GEN) (purity 96.5\%), Tobramycin (TOB) (purity 93\%), Streptomycin sulphate (STR) (purity 98\%), Dihydrostreptomycin sesquisulfate hydrate (DIH) (purity 99\%), Spectinomycin dihydrochloride hydrate (SPE)(purity 99\%), Paromomycin sulphate (PAR) (purity 90\%) and Neomycin sulphate (NEO) (purity 90\%) were purchased from Dr. Ehrenstorfer GmbH (Augsburg-Germany). Sisomycin sulphate salt (SIS) (purity 87\%), Kanamycin sulphate (KAN) (purity 77\%), Amikacin Hydrate (AMK) (purity 98\%) and Hygromycin B (HYG) (purity 66\%) were purchased from Sigma-Aldrich (St. Louis, MO).

Water, acetonitrile (ACN) for LC-MS CHROMASOLV and pentafluoropropionic acid (PFPA) (purity 97\%) were obtained from Sigma-Aldrich (GmbH Riedstr steinheim, Germany). Ammonium acetate (AmAc) and trifluoroacetic acid (TFA) were from MERCK (Darmstadt, Germany) and formic acid (FA) from BDH laboratory (England).

\subsection{Preparations of Standard Solutions}

Due to the high sorption affinity of the AGs to polar surfaces, only laboratory equipments made of polypropylene were used during sample preparation and storage. The use of organic acid, such as 1\% FA in stock solution allows the adsorption to plastic tubes.

Stock standard solutions preparations $\left(\sim 1000 \mu \mathrm{g} \cdot \mathrm{mL}^{-1}\right)$ : $0.02 \mathrm{~g}$ of each AG were dissolved in $25 \mathrm{~mL}$ volumetric flask and then reconstituted with water at $1 \% \mathrm{FA}$. The solutions were stored at $4{ }^{\circ} \mathrm{C}$ and were stable for at least 8 months. For the optimization of MS/MS parameters (tuning), an individual standard solution was prepared at $5 \mu \mathrm{g} \cdot \mathrm{mL}^{-1}$ in ACN-Water $(1: 1, \mathrm{v} / \mathrm{v})$. For the standard calibration curves, a mixture of standard solution at highest concentration $\left(35 \mu \mathrm{g} \cdot \mathrm{mL}^{-1}\right)$ was prepared in water at $1 \% \mathrm{FA}$. This solution was then appropriately diluted to lower concentrations. 


\subsection{Mass Spectrometer Conditions}

Analysis was applied using HPLC from Agilent (Agilent technologies, USA) series 1200 coupled with MS/MS triple quadruple Agilent 6410. The Agilent HPLC was equipped with an automatic degasser, a quaternary pump, a cooled autosampler and an ESI ion source. The optimization was carried out by infusing $5 \mu \mathrm{g} \cdot \mathrm{mL}^{-1}$ of each standard in the HPLC mobile phase into the MS/MS system for automatic tuning to achieve the maximum response. Then, MS/MS data acquisition was performed in Multiple Reaction Monitoring (MRM) mode where two precursor-product ions were chosen for each AGs and listed in Table 1. The precursor ions of all analytes were $[\mathrm{M}+\mathrm{H}]^{+}$, except SPE, which forms intensive water adducts. SPE has an unusual structural feature in which the carbonyl group is hydrated in an aqueous solution [24]. Therefore, $\left[\mathrm{M}+\mathrm{H}_{2} \mathrm{O}+\mathrm{H}\right]^{+}, \mathrm{m} / \mathrm{z} 351$ was selected as the precursor ion for MS/MS analysis.

The MS/MS instrument was operated in positive ion mode using nitrogen gas as a collision gas and also as nebulization gas at 30 and 40 psi respectively. The source temperature was maintained at $350^{\circ} \mathrm{C}$ and capillary voltage was set at $4 \mathrm{kV}$. The fragment ions, the optimized cone voltage and the collision energy are shown in Table 1. Fragment ions with the highest intensity were chosen for quantification and the dwell time was fixed at 25.

\subsection{Chromatographic Separation}

Two types of LC conditions were applied for the analysis of the 12 AGs: Ion pairing chromatography and HILIC. To optimize chromatographic separation, preliminary experiments were performed using both methods.

\subsection{ZIC_HILIC Conditions}

Separation of AGs was performed on a SeQuant ZIC_HILIC PEEK column $50 \mathrm{~mm} * 2.1 \mathrm{~mm}, 3.5 \mu \mathrm{m}$ particle sizes. The column was contained in a thermostated column oven maintained at $30^{\circ} \mathrm{C}$. Mobile phases were: AUltrapure water with $30 \mathrm{mM} \mathrm{AmAc}+\mathrm{ACN}+\mathrm{TFA}(95 / 5 / 0.1)$ and B: Ultrapure water with $2 \mathrm{mM} \mathrm{AmAc}+\mathrm{ACN}$ + TFA (5/95/0.2). The elution gradient program (A: B) was applied: (0:100) to (70:30) in 2 min at $0.3 \mathrm{~mL} \cdot \mathrm{min}^{-1}$; (70:30) to (95:5) in $1 \mathrm{~min}$ at $0.3 \mathrm{~mL} \cdot \mathrm{min}^{-1}$ : (95:5) to (5:95) in $1 \mathrm{~min}$ at $0.5 \mathrm{~mL} \cdot \mathrm{min}^{-1}$; (5:95) to $(0: 100)$ in $1 \mathrm{~min}$ at $0.3 \mathrm{~mL} \cdot \mathrm{min}^{-1}$. Then, the column was equilibrated for $5 \mathrm{~min}$ until the next injection with the flow rates 0.3 $\mathrm{mL} \cdot \mathrm{min}^{-1}$; the total run time of the method was $19 \mathrm{~min}$. An injection volume of $10 \mu \mathrm{L}$ was employed for all samples tested.

\subsection{Ion Pairing Chromatography}

A suitable reversed phase separation of a mixture of AGs was achieved with a $150 \mathrm{~mm} * 4.6 \mathrm{~mm} 5 \mu \mathrm{m}$ Zorbax Eclipse C18 column. Mobile phases were: A-Ultrapure water + 0.1\% PFPA and B: ACN. The elution gradient

\section{Table 1. The optimal parameters for multiple reaction monitoring (MRM) for 12 AGs.}

\begin{tabular}{|c|c|c|c|c|c|c|}
\hline Aminoglycosides & $\begin{array}{l}\text { Precursor } \\
\text { ion }(m / z)\end{array}$ & $\begin{array}{c}\text { Cone } \\
\text { voltage }(\mathrm{V})\end{array}$ & $\begin{array}{l}\text { Quantification } \\
\text { ion }(\mathrm{m} / \mathrm{z})\end{array}$ & $\begin{array}{c}\text { Collision } \\
\text { energy (V) }\end{array}$ & $\begin{array}{l}\text { Confirmation } \\
\text { ion }(\mathrm{m} / \mathrm{z})\end{array}$ & $\begin{array}{c}\text { Collision } \\
\text { energy (V) }\end{array}$ \\
\hline Apramycin & 540.3 & 90 & 217 & 25 & 378 & 15 \\
\hline Gentamycin & $\begin{array}{l}478.3 \\
464.3 \\
450.1\end{array}$ & 120 & $\begin{array}{c}322.2 \\
322.1 \\
322\end{array}$ & 10 & 156.9 & 20 \\
\hline Dihydrostreptomycin & 584.3 & 120 & 262.9 & 30 & 245.9 & 35 \\
\hline Streptomycin & 582.2 & 120 & 263.1 & 35 & 245.9 & 35 \\
\hline Néomycin & 615.3 & 120 & 160.9 & 35 & 292.9 & 25 \\
\hline Spectinomycin & 351.1 & 120 & 333.1 & 15 & 207.1 & 20 \\
\hline Paromomycin & 616.3 & 100 & 162.9 & 40 & 324.1 & 20 \\
\hline Tobramycin & 468.2 & 120 & 162.9 & 20 & 324.2 & 10 \\
\hline Amikacin & 586.3 & 110 & 162.9 & 35 & 425.3 & 15 \\
\hline Sisomycin & 448.3 & 100 & 254.1 & 15 & 271.1 & 15 \\
\hline Kanamycin & 485 & 60 & 324.2 & 15 & 163.2 & 5 \\
\hline Hygromycin & 528.2 & 110 & 177 & 30 & 352.1 & 25 \\
\hline
\end{tabular}


program (A:B) was applied: (95:5) to (50:50) in $10.6 \mathrm{~min}$; $(50: 50)$ to $(0: 100)$ in $0.4 \mathrm{~min}$; $(0: 100)$ to $(95: 5)$ in 3 min. Then, the column was equilibrated for 3 min until the next injection; the total run time of the method was $16 \mathrm{~min}$ using $0.8 \mathrm{~mL} \cdot \mathrm{min}^{-1}$. A $10 \mu \mathrm{L}$ injection volume was also used in this method.

\section{Results and Discussion}

\subsection{HILIC-ESI-MS/MS Optimization}

Recently, a HILIC phase prepared by graft polymerization to incorporate 3-sulfopropyl dimethylalkyl ammonium inner salts, i.e. sulfoalkylbetaine functional groups onto silica and polymer particles has released. Sometimes, this phase is called a zwitterionic phase. These types of columns are available from SeQuant (Ume, Sweden), as ZIC_HILIC columns. Originally, this phase was prepared for cation exchange chromatography, and earlier reports described the separations of inorganic salts and proteins in fully aqueous mobile phases [25]. This phase can retain not only polar and charged compounds, but it also interacts with neutrally charged analytes (containing either positive or negative charges), increasing selectivity in the analysis of a wide range of compounds. This phase is widely accepted by many researchers, and used often in more recent publications [26].

As mentioned in Section Introduction, highly polar compounds are not retained and are eluted in the void volume of column when using reversed-phase separation. Polar analytes can be more strongly retained in the HILIC mode and are eluted by increasing the percentage of aqueous portion in the mobile phase. The stationary HILIC phase described in literature is a zwitterionic silica gel. Therefore, the HILIC technique is suitable for the analysis of polar compounds (e.g. folates [27], carbohydrates [28], peptides [29] or natural products [30]) with MS/MS detection. The separation mechanism of HILIC is opposite to that of reversed phase liquid chromatography (RPLC). Using a solvent with $95 \% \mathrm{ACN}$, a complete retention of the hydrophilic analytes was observed for more than $20 \mathrm{~min}$. Otherwise, when using a solvent with $90 \%$ water, the hydrophilic analytes were eluted with the front [31].

A wide variety of HILIC columns are commercially available with different functionalities of stationary phase and other features (ZIC_HILIC, Acquity UPLC BEH HILIC...) [32]. Mobile phase composition is an additional important consideration in LC-ESI-MS/MS, especially for trace analysis, in order to achieve high ionization and sufficient separation with minimum matrix interference [32].

Ishii. R et al. found that ZIC_HILIC column was better than other columns in separating the AGs and resolving the corresponding peak form. According to their work, ACN alone was better as a mobile phase than methanol $(\mathrm{MeOH})$ or a mixture of ACN /MeOH $(1: 1, \mathrm{v} / \mathrm{v})$.

In HILIC, the presence of water in the mobile phase is fundamental for the establishment of a stagnant enriched aqueous layer on the surface of the stationary phase into which analytes may selectively be partitioned. The use of a buffered mobile phase is crucial in order to achieve acceptable repeatability for the LC separation of charged compounds, since electrostatic interactions between the solute and the stationary phase are controlled by the buffer. The concentration of the buffer should be low to avoid ionization suppression in the ESI.

Using zwitterionic ZIC_HILIC columns, many problems were observed with the constancy of the retention times and backpressure after 250 samples. Analytes injected on new columns have up to $100 \%$ longer retention times than the same analytes after 20 injections on this column. To remove remaining organic solvent and polar impurities, $20 \mu \mathrm{L}$ of $0.5 \mathrm{M}$ sodium chloride solution were injected after 15 samples. If the backpressure increases or a shift in the selectivity is observed, an initial washing with deionised water is required to remove organic solvent and polar impurities, followed by a flush with $0.5 \mathrm{M}$ sodium chloride solution. Removing salt solution with sufficient water is recommended and finally the column is filled with $80 \%$ ACN [31].

The effect of acid and salts added to the mobile phase for accelerating and stabilizing the ionization, was examined. AGs are eluted from HILIC columns starting with a high concentration of AmAc. It seems necessary to use concentrated AmAc in the mobile phase for eluting all the tested compounds from the analytical column, especially NEO, APR and GEN. In order to investigate the efficiency of ZIC_HILIC, we also designed experiments to compare the chromatographic responses of 12 AGs. Furthermore, mobile phase gradient conditions were also optimized in order to obtain the best chromatographic results with minimum analysis time. Therefore, we presented and discussed the effect of the percentage of organic solvent (ACN), the concentration of AmAc at the starting time, the percentage and the acid type used in the mobile phase, on the separation response area and the corresponding peak form of each compound. All experiment was performed in triplicate and the RSD were in the range $1 \%-20 \%$. 


\subsection{Effect of Acetonitrile on Aminoglycoside Retention}

The effect of ACN on analyte retention was investigated. The experiment demonstrated that, as the percentage of ACN in the starting gradient increased, the retention also increased. Above $60 \%$ ACN, most of the analytes appear to be retained. The organic modifier/aqueous portion ratio is the predominant factor in providing sufficient retention in HILIC. In this issue, the peak of GEN, PAR, NEO, SIS, TOB and APR has a broad and poor form when the percentage of ACN decreases. Thus, this result is related to the decreases of the AmAc concentration (Phase B) and the strong elution with the high percentage of water.

Hence, our optimized gradient started with 95\% ACN then after 2 min the percentage decreases. In addition, the flow $\left(0.3 \mathrm{~mL} \cdot \mathrm{min}^{-1}\right)$ in the starting gradient with $95 \%$ ACN ensured a good retention and separation for all AGs (Figure 2). Furthermore, the effect of ACN on the ending gradient was much less influential as the one in the starting gradient.

\subsection{Effect of Ammonium Acetate Concentration on AGs Response}

The mobile phase in HILIC separation plays important roles and its $\mathrm{pH}$ conditions are generally controlled by buffer solutions with a 10 - $65 \mathrm{mM}$ salt concentration. With respect to the MS sensitivity, the AmAc was superior to the ammonium formate. Therefore, the AmAc was used in different concentrations to obtain sharp peaks and to achieve good responses for all AGs.

Figure 3 shows that the optimum concentration of AmAc was found at $30 \mathrm{mM}$ except that of HYG and AMK. Once the concentration of AmAc increased, the area of majority of AGs was decreased. That's because increasing the concentration of AmAc induced an increase of the ionic strength in the mobile phase. As a consequence, the response of AGs was highly influenced and decreased. This is related to the compound properties. The high ionic strength in the mobile phase lead to discharges and poor spray performance of ionization by ESI.

The response of DIH, STR and GEN did not show a considerable change upon decreasing the concentration of AmAc.

Another fact assumed that the form of peaks of most AGs was improved by increasing AmAc concentration. The late eluted AGs (APR, PAR, SIS, TOB) showed broad peaks when the concentration of AmAc was decreased. This led to a poor limit of detection for this compound. On the other hand, the retention times were increased by raising the concentration of the buffer.

Neomycin has a bad response and a broad peak in ZIC_HILIC column. Hence, many researchers found a great need to add 120 - $150 \mathrm{mM}$ AmAc and a 1\% FA in the mobile phase [1]. However, this was impossible to be done in our work as it negatively influenced the response of other AGs.

\subsection{Effect of Percentage and Type of Acid in the Mobile Phase on the Ionization for AGs}

The effect of three different percentages of FA (0.1, 0.2 and 0.5$)$ added to the mobile phase A was studied. We found that the retention times were not affected when varying the $\mathrm{pH}$ of the mobile phase. This indicates the high selectivity and the $\mathrm{pH}$ independence of all compounds. Most of AGs showed an increase in response when changing the percentage of FA from 0.5 to 0.1 . So the percentage $0.1 \%$ (pH 4.30) was shown to be better than $0.2 \%(\mathrm{pH} 3.51)$ and $0.5 \%(\mathrm{pH} 3.22)$ (Figure 4). However, GEN, DIH, STR presented an exception as their response increase when $0.5 \%$ FA was used (Figure 4).

Another experiment studied the effect of response for all AGs substituting FA by TFA. When using $0.1 \%$ TFA ( $\mathrm{pH}$ 4.88), the results presented in the Figure 5 showed an improvement in the peak area for majority of AGs (AMK-HYG-KAN-NEO-APR-GEN-PAR-SIS-TOB) to the detriment of 0.1\% FA (pH 4.30). In contrast, the remaining AGs (SPE-DIH-STR) revealed an improvement in the response when using 0.1\% FA.

\subsection{Ion Pairing Optimization}

Most of previously reviewed methods are mainly based on reversed phase separation due to its applicability for a wide range of neutral compounds of different polarities. However, the main drawbacks encountered when using reversed phase for the most highly polar compounds, that they are poorly retained and produce poor peak shapes. Hammel et al. [33] proposed ion pair chromatography for multiresidue antibiotics analysis in honey, including AGs. However, ion pair separation requires ion-pair reagent (mainly TFA, heptafluorobutyric acid, phosphoric acid, etc.) in the mobile phase composition to regulate the retention of polar compounds on the stationary phase [32]. 

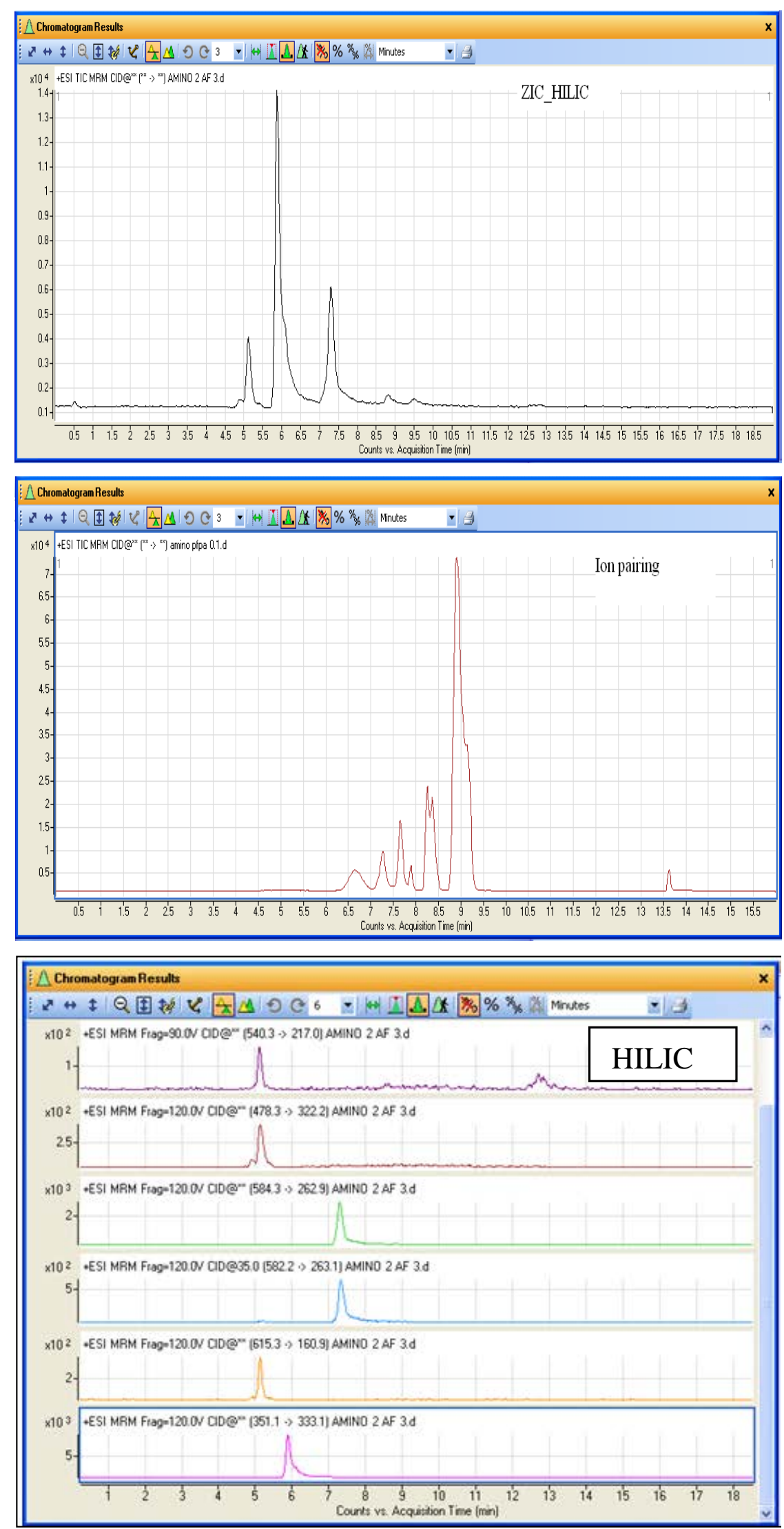


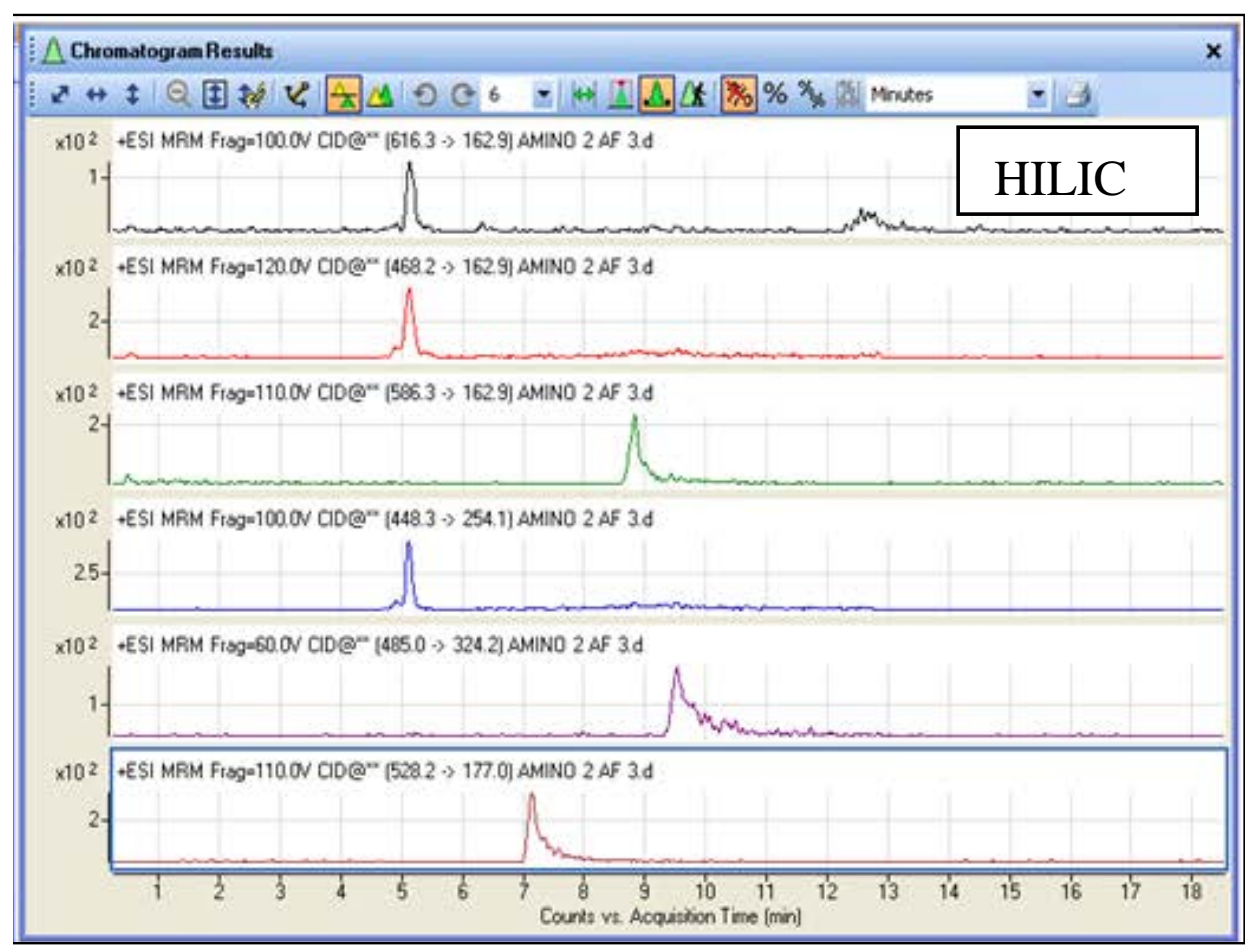

Figure 2. TIC obtained for the 12 AGs by the two methods of separation (HILIC and Ion Pairing) at a $100\left(\mu \mathrm{g} \cdot \mathrm{mL}^{-1}\right)$ and chromatogram obtained at $95 \%$ ACN (starting gradient) in ZIC-HILIC column.

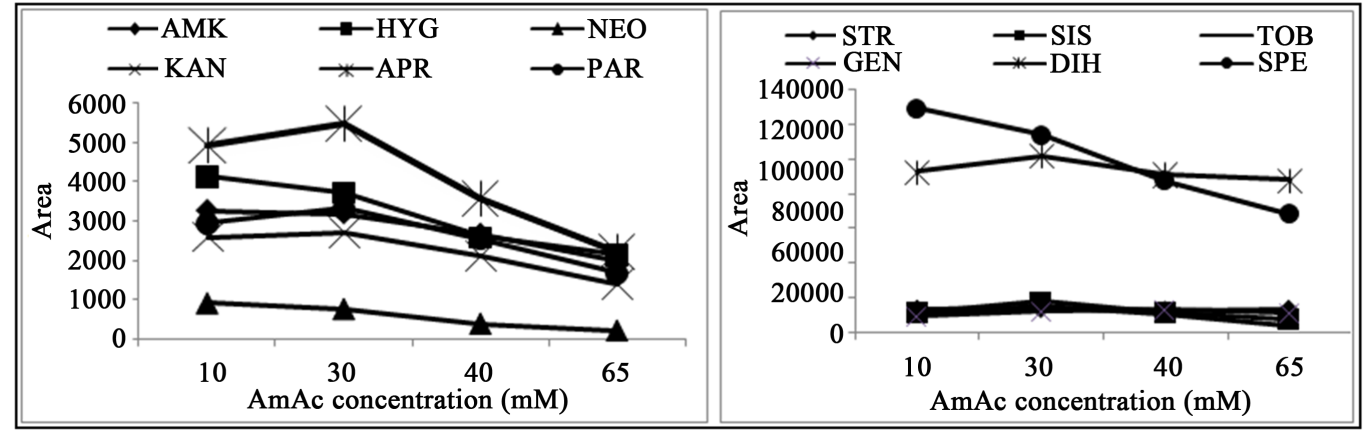

Figure 3. Effect of the buffer (ammonium acetate AmAc) concentration in the mobile phase A on the response of the 12 AGs.

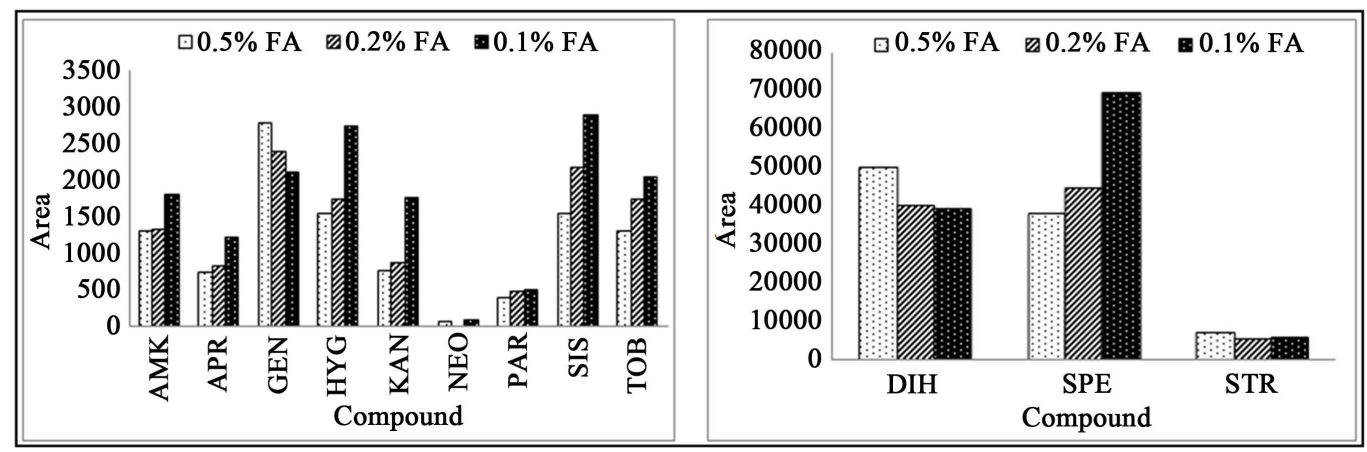

Figure 4. Effect of the percentage of Formic acid $(0.5 \%, 0.2 \%$ and $0.1 \%)$ in the mobile phase A on the response of AGs. 


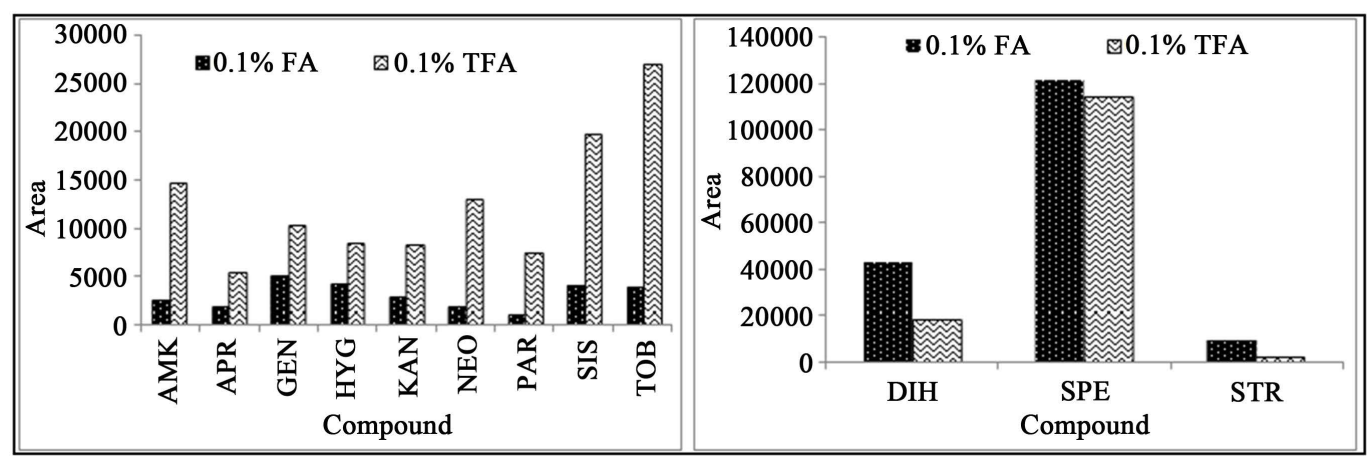

Figure 5. Effect of the acid type, TFA and FA, in the mobile phase A at $0.1 \%$ on the response of all AGs.

Therefore, some volatile and fluorinated ion-pair reagents including TFA, PFPA and heptafluorobutyric acid (HFBA) are commonly used in the previously published methods for the analysis of AGs combined with mass spectrometry [17] [23] [34].

When HFBA and PFPA are compared, PFPA can be considered as a better reagent, because it does not adsorb to the stationary phase as HFBA strongly does and the retention is clearly obtained through an ion-pair mechanism [35]. On the other hand, with TFA as an ion pair agent, dramatic ion suppression in the ESI occurs. Whereas with HFBA the ion suppression was limited [9] [22].

In our work, the PFPA was used as an ion pairing reagent. Their concentration was optimized to get less signal suppression in the ESI. Therefore, the maximum concentration of additives that can be used depends on the design and technical solutions of the ion-source of the mass spectrometric instrument, e.g. orthogonal electrospray instruments can often tolerate higher concentration of additives [36]. Figure 6 presents the comparison of response by using modified concentrations of PFPA $\left[0.92 \mathrm{mmol} \cdot \mathrm{L}^{-1}(0.01 \%), 9.20 \mathrm{mmol} \cdot \mathrm{L}^{-1}(0.1 \%), 46\right.$ $\left.\mathrm{mmol} \cdot \mathrm{L}^{-1}(0.5 \%)\right]$ in the mobile phase. This parameter was found in triplicate and the RSD was between $1 \%$ $12 \%$.

All compound's areas present an optimum result at $0.1 \%$ PFPA. The variation of the results of the peak areas for AGs was designed between $18 \%-80 \%$ when comparing $0.1 \%$ and $0.5 \%$ PFPA. This result became logic if one takes into account the suppression of signal in the ion source (ESI) with the use of high concentration of ion pair reagent. On the other hand, the area response of AGs was better when $0.1 \%$ PFPA was used at the expense of $0.01 \%$ PFPA. The deviation of response was $5 \%$ to $77 \%$. Consequently, $0.01 \%$ PFPA is not sufficient to ensure the optimum retention of analyte in the column.

\subsection{Comparison between HILIC and Ion Pairing}

AGs are very hydrophilic compounds and are traditionally difficult to retain on conventional HPLC columns. Hence, ion-pair reversed phase LC is preferred to chromatograph aminoglycosides on a C18 column. The use of an ion-pairing reagent in the mobile phase has been reported earlier [5] [22] [23] [37]. On the other hand, the application of HILIC is known to be as an alternative for the determination of extremely polar compounds. Recently, literature and research on hydrophilic interaction chromatography (HILIC) have been increasing drastically, along with various stationary phases developed. HILIC is a kind of normal-phase liquid chromatography (NPLC), and has attracted the attention of researchers that study the separation of polar compounds in a wide variety of scientific fields. In HILIC mode, a mixture of water and organic modifiers (mostly ACN) is employed with a polar stationary phase. Structural variations in HILIC type stationary phases are wider than those found in reversed-phase applications. This technique is suitable for ESI-MS, because of the compatibility of the aqueous organic mobile phase to ESI-MS, which is a very powerful tool in detecting and identifying a wide range of polar compounds [26].

The confirmation and quantification of AGs by LC-MS/MS was evaluated by ZIC_HILIC and ion pairing separation. As seen in Figure 2, the ion pairing separation was found better for most of AGs. The response by ion pair reagent showed an increase of the obtained value with ZIC_HILIC conditions (Figure 7). An exception of DIH, STR and SPE, the area was improved in HILIC than in ion pair mode. This led to higher S/N ratios and low limits of detection for the method using ion pairing separation. 


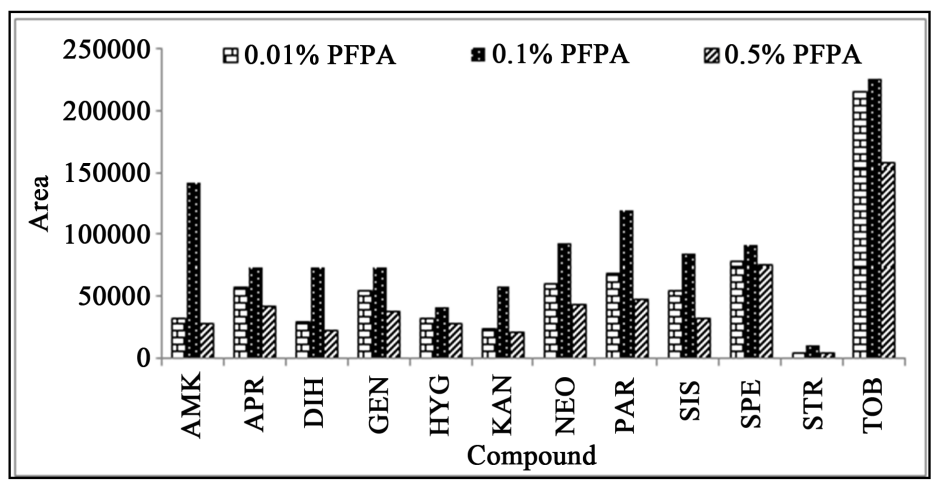

Figure 6. The area response for AGs using 3 different percentages $(0.01 \%$, $0.1 \%$ and $0.5 \%$ ) of PFPA.

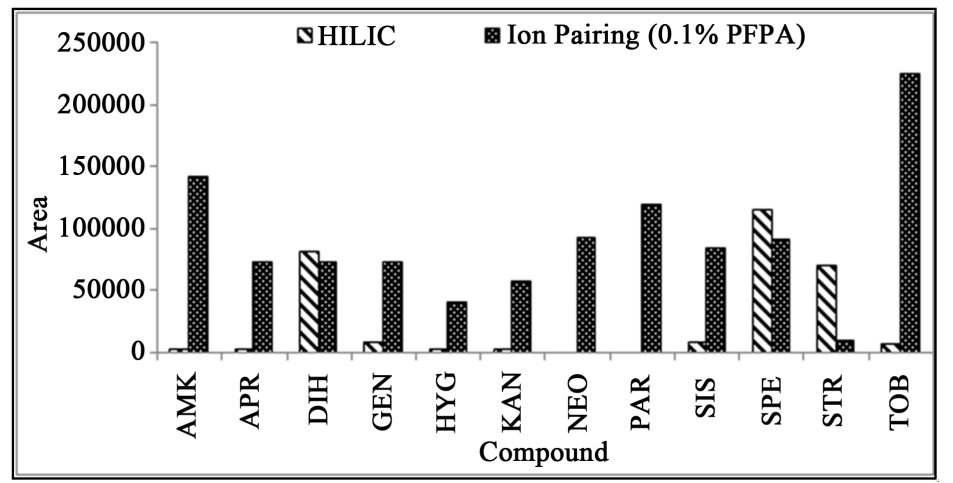

Figure 7. Comparison of area response of AGs by the two methods of separation (HILIC and Ion pairing).

Repeatability of the two separation methods were described as the value of relative standard deviation (RSDs) of the areas obtained for each analyte after the replicate $(n=3)$ analyses of spiked water blank samples which ranged between $0.5 \%-20 \%$. LODs were experimentally determined at a signal to noise ratio $(\mathrm{S} / \mathrm{N})$ of 3 and presented for each AGs. Then, Table 2 present the calculated values (at the same concentration $700 \mu \mathrm{g} \cdot \mathrm{mL}^{-1}$ ) of $\mathrm{S} / \mathrm{N}$ and the limit of detection of the two methods of separation used in our work and certified results was cited above. Linearity of the two methods (HILIC, Ion pairing) compared was determined by injection of blank water samples spiked at different concentration levels ranged between 5 to $500 \mu \mathrm{g} \cdot \mathrm{L}^{-1}$. Each compound showed good linearity in studied working range, with coefficient of determination $\left(\mathrm{R}^{2}\right)$ greater than 0.98 (Table 2). The best coefficient of determination (0.982 - 0.998) was related to Ion pairing method for the majority of AGs.

ZIC_HILIC columns need mobile phases with high organic content. This type of column gives large peak areas when used without modifiers, but they required high concentrations of AmAc to achieve reasonable peak shapes. But, the high concentration of AmAc greatly suppressed the response from the mass spectrometer [22].

Another problems were found, the ZIC_HILIC column need more time for conditioning in comparison to the C18 column by using PFPA reagent. This fact was demonstrated by the injection of standards several times at the beginning of conditioning. The better result of separation by ZIC_HILIC was performed for each AG after 1 $\mathrm{h}$ conditioning.

Moreover, the column of ZIC_HILIC needs a wash for several hours by water and $0.5 \mathrm{mM} \mathrm{NaCl}$ solution in order to eliminate the back pressure after 250 injection samples. As a consequence, the use of ion pairing modifiers and reversed phase for $L C$ was preferred over HILIC. The ion pair system was showed to be stable after two to three injections. It produced significantly higher sensitivities for late eluting AGs than HILIC and was very stable regarding the injection of high salt containing extracts.

The PFPA reagent was used to regulate the retention of AGs on the C18 column. But many researchers reported the serious problem of the affected MS performance by ionization suppression and the contamination of the ion source when using the ion pair reagent [32]. 
Table 2. Coefficients of determination $\left(\mathrm{R}^{2}\right)$ for the calibration curves signal to noise $(S / N)$ ratio, Limit of detection $\left(\mu \mathrm{g} \cdot \mathrm{min}^{-1}\right)$ and retention times (Tr) of the two methods (HILIC and Ion Pairing).

\begin{tabular}{|c|c|c|c|c|c|c|c|c|}
\hline \multicolumn{5}{|c|}{ HILIC } & \multicolumn{4}{|c|}{ Ion pairing } \\
\hline Aminoglycosides & $\mathrm{R}^{2}$ & LOD (ppb) & $\mathrm{S} / \mathrm{N}$ & $\operatorname{Tr}(\min )$ & $\mathrm{R}^{2}$ & LOD (ppb) & $\mathrm{S} / \mathrm{N}$ & $\operatorname{Tr}(\min )$ \\
\hline Amikacin & 0.991 & 10 & 3.12 & 9.7 & 0.998 & 5 & 148 & 8.2 \\
\hline Apramycin & 0.988 & 90 & 3.96 & 5.3 & 0.991 & 5 & 138 & 8.8 \\
\hline Dihydrostreptomycin & 0.996 & 5 & 11.4 & 5.4 & 0.994 & 5 & 57.4 & 7.2 \\
\hline Gentamicin & 0.989 & 60 & 4.99 & 5.3 & 0.989 & 5 & 204 & 9.1 \\
\hline Hygromycin & 0.994 & 5 & 8.08 & 7.2 & 0.949 & 5 & 10 & 7.2 \\
\hline Kanamycin & 0.995 & 90 & 3 & 10.2 & 0.982 & 5 & 138 & 8.3 \\
\hline Neomycin & 0.420 & 200 & 4.05 & 5.3 & 0.994 & 5 & 161 & 9.3 \\
\hline Paromomycin & 0.981 & 200 & 3.1 & 5.1 & 0.991 & 5 & 277 & 8.8 \\
\hline Sisomycin & 0.979 & 200 & 2.10 & 5.2 & 0.990 & 5 & 168 & 9 \\
\hline Spectinomycin & 0.942 & 5 & 52 & 6.7 & 0.979 & 15 & 12.6 & 6.7 \\
\hline Streptomycin & 0.987 & 5 & 9.06 & 5.4 & 0.993 & 15 & 17.6 & 9.1 \\
\hline Tobramycin & 0.968 & 200 & 3.4 & 5.3 & 0.987 & 5 & 169 & 8.9 \\
\hline
\end{tabular}

Sometimes, it is reported that these reagents adhere to the line on $L C$ and $M S$ detectors, which reduces the MS sensitivity and requires maintenance of $L C$ and $M S$ equipment due to these contaminants [38].

Finally, regarding all the results and the problems cited above, the separation by reversed phase C18 using the PFPA is more suitable than the separation by ZIC_HILIC. Yet, cleaning the instrument was not found to be a relevant problem when using the ion pair reagent.

\section{Conclusions}

Two types of separation conditions (HILIC, Ion pairing) were examined and optimized to improve the chromatographic parameters for the analysis of 12 aminoglycosides by LC-MS/MS.

In ZIC_HILIC column, different concentrations of ammonium acetate and formic acid have been tested. As a result, $30 \mathrm{mM}$ ammonium acetate and $0.1 \% \mathrm{FA}$ appear to induce better conditions for most analytes. As regards to the pentafluoropropionic acid (PFPA), an optimum concentration was evaluated for ion pairing using Zorbax Eclipse C18 column $\left(9.2 \mathrm{mM} \cdot \mathrm{L}^{-1}\right)$. The advantages and the disadvantages of the two methods were discussed and reported. Both ion pairing and HILIC, demonstrated good separation of the majority of compounds tested.

When comparing the response area of HILIC and ion pairing, most of the AGs showed an increase of around 99\% by ion pairing. However, Dihydrostreptomycin, Streptomycin and Spectinomycin showed better area responses when separation was made with HILIC. Both limit of detection and $\mathrm{S} / \mathrm{N}$ ratio were better when using ion pairing. On the other hand, the problems for the constancy of the retention times and the backpressure in the column make the ion pairing a reliable and powerful technique comparing to HILIC.

\section{Acknowledgements}

We thank the Lebanese National Council for Scientific Research (CNRSL) and the Lebanese Atomic Energy Commission (LAEC) for technical support.

\section{References}

[1] McGlinchey, T., Rafter, P., Regan, F. and McMahon, G.A. (2008) Review of Analytical Methods for the Determination of Aminoglycoside and Macrolide Residues in Food Matrices. Analytica Chimica Acta, 624, 1-15. http://dx.doi.org/10.1016/j.aca.2008.05.054

[2] Stevens, R.C. and Rodman, J.H. (1998) Pharmacokinetics of Antimicrobial Therapy. Seminars in Pediatric Infectious 
Diseases, 9, 273-280. http://dx.doi.org/10.1016/S1045-1870(98)80016-2

[3] Turnipseed, S.B. and Long, A.R. (1998) Analytical Procedures for Drug Residues in Food of Animal Origin. Science Technology System.

[4] Shulman, E., Belakhov, V., Wei, G., Kendall, A., Meyron-Holtz, G., Ben-Shachar, D., Schacht, J. and Baasov, T. (2013) Designer Aminoglycosides That Selectively Inhibit Cytoplasmic rather than Mitochondrial Ribosomes Show Decreased Ototoxicity: A Strategy for the Treatment of Genetic Diseases. The Journal of Biological Chemistry. http://dx.doi.org/10.1074/jbc.M113.533588

[5] Zhu, W., Yang, J., Wei, W., Liu, Y. and Zhang, S. (2008) Simultaneous Determination of 13 Aminoglycoside Residues in Foods of Animal Origin by Liquid Chromatography-Electrospray Ionization Tandem Mass Spectrometry with Two Consecutive Solid-Phase Extraction Steps. Journal of Chromatography A, 1207, 29-37. http://dx.doi.org/10.1016/j.chroma.2008.08.033

[6] Commission Decision 2002/657/EC (2002) Performance of Analytical Methods. Official Journal of European Commission. http://eur-lex.europa.eu/legal-content/EN/TXT/?uri=CELEX:32002D0657

[7] Oertel, R., Renner, U. and Kirch, W. (2004) Determination of Neomycin by LC-Tandem Mass Spectrometry Using Hydrophilic Interaction Chromatography. Journal of Pharmaceutical and Biomedical Analysis, 35, 633-638.

[8] Megoulas, N. and Koupparis, M. (2005) Development and Validation of a Novel HPLC/ELSD Method for the Direct Determination of Tobramycin in Pharmaceuticals, Plasma and Urine. Analytical and Bioanalytical Chemistry, 382, 290-296. http://dx.doi.org/10.1007/s00216-004-2948-8

[9] Garcia-Mayor, M., Garcinuno, M., Fernandez-Hernando, P. and Durand-Alegria, J. (2006) Liquid Chromatography-UV Diode-Array Detection Method for Multi-Residue Determination of Macrolide Antibiotics in Sheep's Milk. Journal of Chromatography A, 1122, 76-83. http://dx.doi.org/10.1016/j.chroma.2006.04.019

[10] Serrano, M. and Silva, M. (2006) Determination of Amikacin in Body Fluid by High-Performance Liquid-Chromatography with Chemiluminescence Detection. Journal of Chromatography B, 843, 20-24. http://dx.doi.org/10.1016/j.jchromb.2006.05.016

[11] Abuin, S., Codony, R., Compano, R., Granados, M. and Prat, M.D. (2006) Analysis of Macrolide Antibiotics in River Water by Solid-Phase Extraction and Liquid Chromatography-Mass Spectrometry. Journal of Chromatography A, 1114, 73-81. http://dx.doi.org/10.1016/j.chroma.2006.02.032

[12] Preu, M., Guyot, D. and Petz, M. (1998) Development of a Gas Chromatography-Mass Spectrometry Method for the Analysis of Aminoglycoside Antibiotics Using Experimental Design for the Optimisation of the Derivatisation Reactions. Journal of Chromatography A, 818, 95-108. http://dx.doi.org/10.1016/S0021-9673(98)00537-8

[13] Clarot, I., Chaimbault, P., Hasdenteufel, F., Netter, P. and Nicolas, A. (2004) Determination of Gentamicin Sulfate and Related Compounds by High-Performance Liquid Chromatography with Evaporative Light Scattering Detection. Journal of Chromatography A, 1031, 281-287. http://dx.doi.org/10.1016/j.chroma.2003.12.032

[14] Tawa, R., Matsunaga, H. and Fujimoto, T. (1998) High-Performance Liquid Chromatographic Analysis of Aminoglycoside Antibiotics. Journal of Chromatography A, 812, 141-150. http://dx.doi.org/10.1016/S0021-9673(98)00342-2

[15] Posyniak, A., Zmudzki, J. and Niedzielska, J. (2001) Sample Preparation for Residue Determination of Gentamicin and Neomycin by Liquid Chromatography. Journal of Chromatography A, 914, 59-66. http://dx.doi.org/10.1016/S0021-9673(00)00980-8

[16] Kim, B., Otsuka, K. and Terabe, S. (2001) Anion Selective Exhaustive Injection-Sweep-Micellar Electrokinetic Chromatography. Journal of Chromatography A, 932, 129-137. http://dx.doi.org/10.1016/S0021-9673(01)01246-8

[17] McGlinchey, T. (2010) The Determination of Veterinary Antibiotics in Live Animals and Animal Products. School of Chemical Sciences Dublin City University, Dublin.

[18] Cai, Y.Q., Cai, Y.E., Mou, S.F. and Lu, Y.Q. (2005) Multi-Walled Carbon Nanotubes as a Solid-Phase Extraction Adsorbent for the Determination of Chlorophenols in Environmental Water Samples. Journal of Chromatography A, 1081, 245-247. http://dx.doi.org/10.1016/j.chroma.2005.05.080

[19] Kato, Y., Iwamoto, M., Koike, T., Suzuki, F. and Takano, Y. (1988) Terminal Differentiation and Calcification in Rabbit Chondrocyte Cultures Grown in Centrifuge Tubes: Regulation by Transforming Growth Factor Beta and Serum Factors. Proceedings of the National Academy of Sciences of the United States of America, 85, 9552-9556. http://dx.doi.org/10.1073/pnas.85.24.9552

[20] Sakairi, M. and Kambara, H. (1988) Characteristics of a Liquid Chromatography/Atmospheric Pressure Ionization Mass Spectrometer. Analytical Chemistry, 60, 774-780. http://dx.doi.org/10.1021/ac00159a009

[21] Tao, Y., Chen, D., Yu, H., Huang, L., Liu, Z., Cao, X., Yan, C., Pan, Y., Liu, Z. and Yuan, Z. (2012) Simultaneous Determination of 15 Aminoglycoside(s) Residues in Animal Derived Foods by Automated Solid-Phase Extraction and Liquid Chromatography-Tandem Mass Spectrometry. Food Chemistry, 135, 676-683. http://dx.doi.org/10.1016/j.foodchem.2012.04.086 
[22] Plozza, T., Trenerry, V., Zeglinski, P., Nguyen, H. and Johnstone, P. (2011) The Confirmation and Quantification of Selected Aminoglycoside Residues in Animal Tissue and Bovine Milk by Liquid Chromatography Tandem Mass Spectrometry. International Food Research Journal, 18, 1077-1084.

[23] Loffler, D. and Ternes, T. (2003) Analytical Method for the Determination of the Aminoglycoside Gentamicin in Hospital Wastewater via Liquid Chromatography_Electrospray-Tandem Mass Spectrometry. Journal of Chromatography A, 1000, 583-588. http://dx.doi.org/10.1016/S0021-9673(03)00059-1

[24] McLaughlin, L.G. and Henion, I.D. (1994) Multi Residue Confirmation of Aminoglycosides Antibiotics in Bovine by Ion Spray High Performance Liquid Chromatography/Tandem Mass Spectrometry. Biological Mass Spectrometry, 23, 417-429.

[25] (2008) A Practical Guide to HILIC. Se Quant, Sweden. http://www.mercksequant.com

[26] Ikegami, T., Tomomatsu, K., Takubo, H., Horie, K. and Tanaka, N. (2008) Separation Efficiencies in Hydrophilic Interaction Chromatography. Journal of Chromatography A, 1184, 474-503. http://dx.doi.org/10.1016/j.chroma.2008.01.075

[27] Garbis, S.D., Melse-Boonstra, A., West, C.E. and van Breemen, R.B. (2001) Determination of Folates in Human Plasma Using Hydrophilic Interaction Chromatography-Tandem Mass Spectrometry. Analytical Chemistry, 73, 5358-5364. http://dx.doi.org/10.1021/ac010741y

[28] Alpert, A.J., Shukla, M., Shukla, A.K., Zieske, L.R., Yuen, S.W., Ferguson, M.A., Mehlert, A., Pauly, M. and Orlando, R. (1994) Hydrophilic-Interaction Chromatography of Complex Carbohydrates. Journal of Chromatography A, 676, 191-202. http://dx.doi.org/10.1016/0021-9673(94)00467-6

[29] Oyler, A.R., Armstrong, B.L., Cha, J.Y., Zhou, M.X., Yang, Q., Robinson, R.I., Dunphy, R. and Burinsky, D.J. (1996) Hydrophilic-Interaction Chromatography on Amino-Silica Phases Complements Reversed-Phase High Performance Liquid Chromatography and Capillary Electrophoresis for Peptide Analysis. Journal of Chromatography A, 724, 378383. http://dx.doi.org/10.1016/0021-9673(95)00987-6

[30] Strege, M.A. (1998) Hydrophilic Interaction Chromatography-Electrospray Mass Spectrometry Analysis of Polar Compounds for Natural Product Drug Discovery. Analytical Chemistry, 70, 2439-2445.

[31] Oertel, R., Neumeister, V. and Kirch, W. (2004) Hydrophilic Interaction Chromatography Combined with TandemMass Spectrometry to Determine Six Aminoglycosides in Serum. Journal of Chromatography A, 1058, 197-201. http://dx.doi.org/10.1016/j.chroma.2004.08.158

[32] Chiaochan, C., Koesukwiwat, U., Yudthavorasit, S. and Leepipatpiboon, N. (2010) Efficient Hydrophilic Interaction Liquid Chromatography-Tandem Mass Spectrometry for the Multiclass Analysis of Veterinary Drugs in Chicken Muscle. Analytica Chimica Acta, 682, 117-129. http://dx.doi.org/10.1016/j.aca.2010.09.048

[33] Hammel, Y.A., Mohamed, R., Gremaud, E., LeBreton, M. and Guy, P. (2008) Multi-Screening Approach to Monitor and Quantify 42 Antibiotic Residues in Honey by Liquid Chromatography-Tandem Mass Spectrometry. Journal of Chromatography A, 1177, 58-76. http://dx.doi.org/10.1016/j.chroma.2007.10.112

[34] Confirmation of Aminoglycosides by HPLC-MS/MS (2011) Department of Agriculture Food Safety and Inspection Service Office of Public Health Science, USA. http://www.fsis.usda.gov/wps/wcm/

[35] De Miguel, I., Puech-Costes, E. and Samain, D. (1987) Use of Mixed Perfluorinated Ion Pairing Agents as Solvents in Ion Pair High Performance Liquid Chromatography for the Preparative Purification of Aminoglycoside Antibiotics. Journal of Chromatography A, 407, 109-119. http://dx.doi.org/10.1016/S0021-9673(01)92608-1

[36] Gustavsson, S., Samskog, J., Markides, K. and Langstrom, B. (2001) Studies of Signal Suppression in Liquid Chromatography-Electrospray Ionization Mass Spectrometry Using Volatile Ion-Pairing Reagents. Journal of Chromatography A, 937, 41-47. http://dx.doi.org/10.1016/S0021-9673(01)01328-0

[37] Kaufmann, A., Butcher, P. and Maden, K. (2011) Determination of Aminoglycoside Residues by LC-MS/MS in a Variety of Matrices. Analytica Chimica Acta, 711, 46-53. http://dx.doi.org/10.1016/j.aca.2011.10.042

[38] Ishii, R., Horie, M., Chan, W. and MacNeil, J. (2008) Multi-Residue Quantitation of Aminoglycoside Antibiotics in Kidney and Meat by Liquid Chromatography with Tandem Mass Spectrometry. Food Additives \& Contaminants, 25, 1509-1519. http://dx.doi.org/10.1080/02652030802189740 
Scientific Research Publishing (SCIRP) is one of the largest Open Access journal publishers. It is currently publishing more than 200 open access, online, peer-reviewed journals covering a wide range of academic disciplines. SCIRP serves the worldwide academic communities and contributes to the progress and application of science with its publication.

Other selected journals from SCIRP are listed as below. Submit your manuscript to us via either submit@scirp.org or Online Submission Portal.
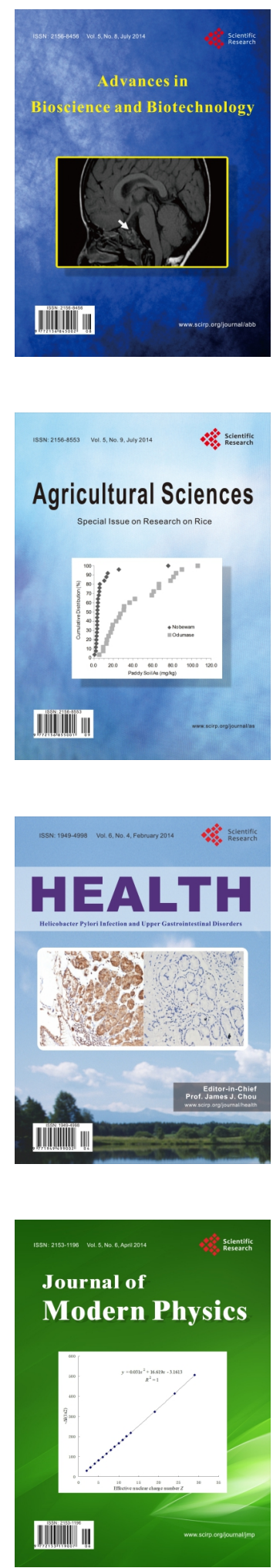
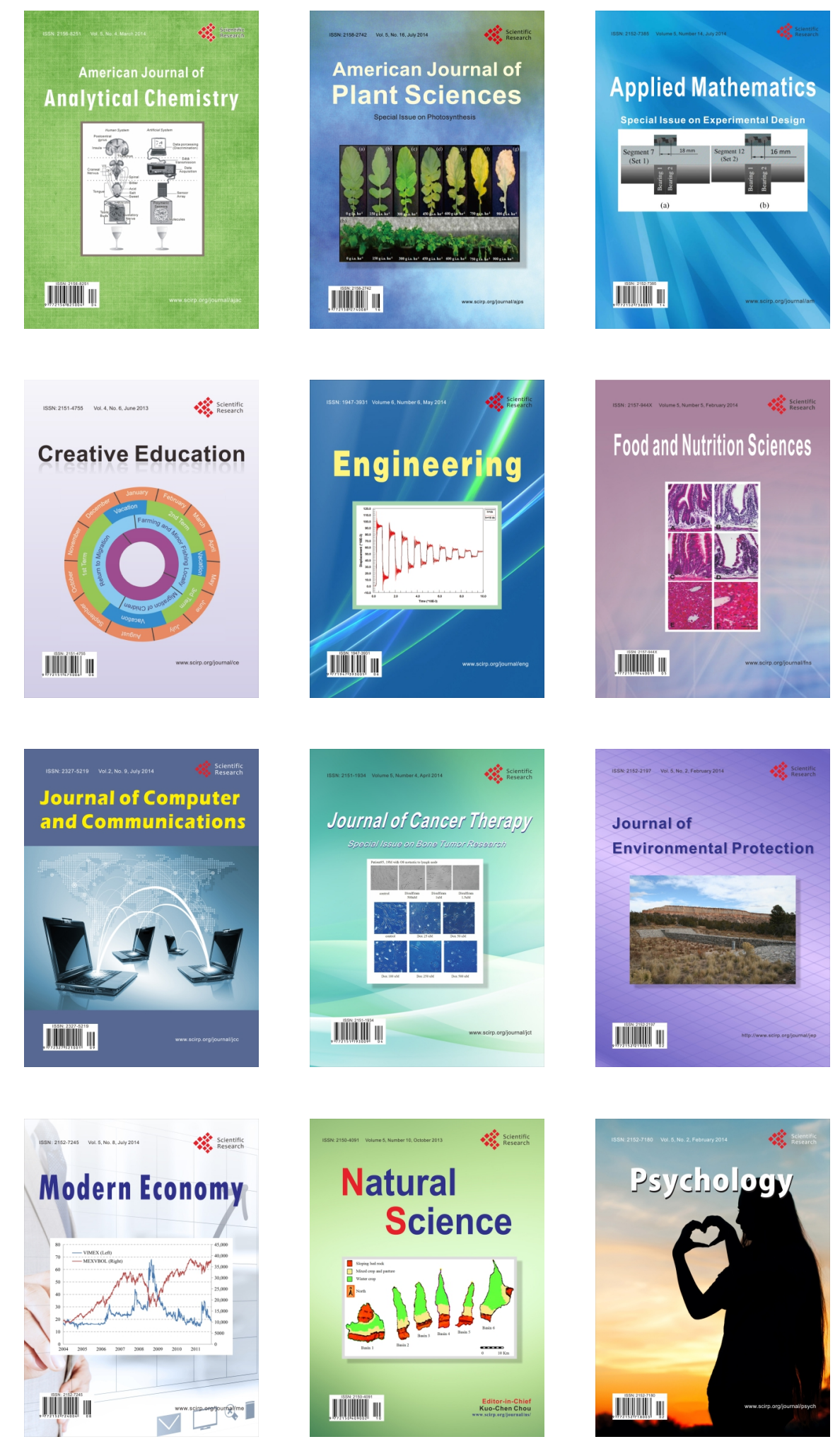